\title{
Whey Protein Isolate-based Nutritional Supplement
}

National Cancer Institute

\section{Source}

National Cancer Institute. Whey Protein Isolate-based Nutritional Supplement. NCI

Thesaurus. Code C158605.

A nutritional supplement composed of a lactose- and gluten-free whey-based protein isolate and containing various vitamins and minerals, with potential immunomodulating activity. In addition to whey protein isolate, this supplement contains phosphoric acid, Lcysteine, ascorbic acid, vitamin E, zinc, ferrous sulfate, niacinamide, vitamin A, calcium pantothenate, copper, manganese, vitamin D3, pyridoxine, thiamine, riboflavin, folic acid, biotin, iodine, phytonadione, and vitamin B12. Upon administration, whey protein isolate is broken down in the body into cysteine and glutamylcysteine. The available cysteine allows cells to synthesize glutathione (GSH), a tripeptide containing amino acids glycine, glutamate and cysteine, thereby maintaining and increasing intracellular GSH concentrations. GSH plays a major role as an antioxidant, thereby protecting cells from oxidative damage due to harmful substances such as free radicals and reactive oxygen compounds. In addition, the supplement provides necessary vitamins and minerals to the body, which may stimulate the immune system, help with wound healing and regulate metabolic dysregulation. 